\section{Converging Technologies und NBIC als Gegenstand von Wissenspolitik?}

\author{
von Christian Büscher, ITAS
}

Seit einigen Jahren werden die wissenschaftspolitischen Themen der "Converging Technologies" (CT) und NBIC (Convergence of Nano-, Bio-, Information Technology and Cognitive Sciences) in sozialwissenschaftlichen und philosophischen Disziplinen diskutiert. Der vorliegende Artikel präsentiert einige Ergebnisse und Interpretationen zu diesem Themenkomplex, die in dem EU-geförderten sozialwissenschaftlichen Projekt „Knowledge NBIC“ generiert wurden. In diesem Artikel werden CT und NBIC mit dem Begriff der "Wissenspolitik“ in Verbindung gebracht und es wird gefragt, welche Aspekte dieser Themen Gegenstand von Politik sein könnten (z. B. bestimmte Formen der „Wissensproduktion") und inwieweit politische "Zugriffe“" möglich erscheinen (z. B. durch das Formulieren von „Förderbedingungen“").

\section{Einleitung}

Hinter den Akronymen NBIC und CT verbergen sich umfangreiche wissenschaftstechnologische Programme mit möglicherweise weitreichenden Folgen (Coenen et al. 2004). Beide Themen haben sich seit Ende der 1990er Jahre hauptsächlich aus dem politischen Diskurs um die Nanotechnologie entwickelt (Coenen 2008, S. 4). Seitdem stehen sie unter Beobachtung einer vieldisziplinären „Begleitforschung", die normative Orientierungen des „Wollens“ mit kognitiven Orientierungen des „Könnens“ abgleichen. Gleichzeitig wurde der Begriff der "Wissenspolitik“ in sozialwissenschaftliche Diskurse eingeführt (Stehr 2003; Bechmann, Stehr 2004; Rammert 2003; Wehling 2006), jedoch jeweils mit unterschiedlichen Konnotationen als Regulierung, als Wissenschafts-, Technologie- und Innovationspolitik oder als „Reflexive Wissenspolitik“. Die specific support action „Knowledge NBIC“ aus dem sechsten EU-Rahmenprogramm hat diesen Begriff als zentrale Orientierung übernommen, um die Entstehung von CT und NBIC als wis- senschaftstechnologische Themen zu explorieren und darauf aufbauend - wie im Folgenden geschehen - um Möglichkeiten der Wissenspolitik zu analysieren. ${ }^{1}$ Dieser Beitrag bespricht einige theoretische Überlegungen und daraus folgende Interpretationen zum Verhältnis von CT/NBIC und Wissenspolitik, die sich im Zuge dieser Exploration ergaben und die für weitere Forschungen fruchtbar gemacht werden sollen. ${ }^{2}$

$\mathrm{Zu}$ diesem Zweck werden an dieser Stelle drei Fragen besprochen. (1) Wie kann mit den zahlreichen Erwartungen, die beide Themen begleiten, sinnvoll umgegangen werden? (2) Welche Annahmen zu dem Begriff von „Wissenspolitik" werden zugrunde gelegt und welche Orientierungen ergeben sich daraus? (3) Inwieweit haben sich CT oder NBIC als „Gegenstand" von Wissenspolitik etabliert und welche Formen der Wissenspolitik lassen sich beschreiben?

\section{Erwartungskomplexe}

Die Idee der „Converging Technologies“ und speziell das Thema NBIC beinhalten die Erwartung, dass unterschiedliche wissenschaftliche Wissensdomänen und Disziplinen konvergieren können. Das bedeutet, dass hypothetisches Wissen von unterschiedlichen Möglichkeiten der Manipulation

- von Bewusstseins-Prozessen (Kognitionswissenschaft),

- von Materie auf Größenordnungen der Nano-Ebene (Nanotechnologie),

- von biologisch-chemische Prozessen von beispielsweise menschlichen Körpern (Biotechnologie), und

zusammengenommen mit Möglichkeiten der rasend schnellen Prozessierung von Algorithmen zur elektronischen Datenverarbeitung (Informationstechnik) "synergetisch“ ein neues wissenschaftstechnologisches Paradigma entstehen lassen könnte. Kurz: „Each of the four research challenges $(. .$.$) focuses on one of the$ NBIC areas (nanotechnology, biotechnology, information technology, and cognitive science) and shows how progress can be catalyzed by convergence with the other areas." (Roco, Bainbridge 2002, S. 13) 
Wie lassen sich nun wissenschaftstechnologische Statements interpretieren, wenn diese offensichtlich die zukünftige Realisierung von Technologien im Blick haben - Technologien, welche umfangreiche und im Sinne der Autoren erwünschte Zwecke erfüllen könnten: human, social and societal enhancement? Wir wollen einige Möglichkeiten besprechen.

a) Keinesfalls können Beurteilungen angefertigt werden, ob die Erwartung der Realisierung nach dem heutigen Stand von Forschung und Technik ,realistisch“ ist. Solche Aussagen bleiben gegebenenfalls den jeweiligen Spezialdisziplinen überlassen.

b) Keinesfalls können Beurteilungen des „Guten“ oder "Schlechten“ der angepeilten Zwecke im Zusammenhang mit der Realisierung der anvisierten Technologien formuliert werden. Bereits Jean-Pierre Dupuy (2004) hat auf grundlegende Missverständnisse in den Diskursen um eine „Nanoethik“ hingewiesen. Ethik würde oftmals mit Vorsicht verwechselt (prudence), also allgemein dem Abwägen zwischen einem möglichen Nutzen einer Technologie und den möglichen Gefahren für Gesundheit, Umwelt, Sicherheit, Privatsphäre usw. Im Grunde genommen handele es sich bei „Nanoethik" oftmals um ein risk-assessment oder um Überlegungen der Vorsorge gegen unerwünschte Folgen. Ethik würde in diesem Sinne zu einem ökonomischen Kalkül reduziert, also in eine Kosten-NutzenRechnung verwandelt, argumentiert Dupuy. Dafür bräuchte man im Allgemeinen akkurate Schadensbeschreibungen, Berechnungen von Wahrscheinlichkeiten hinsichtlich des Eintritts möglicher Schäden sowie Angaben zu allgemeingültigen (geldwerten!) Maßstäben zu Nutzen und Schäden von möglicherweise Betroffenen. Im Hinblick auf den Ausblick auf eine ,transformation of civilization “3 kann sich Dupuy nicht vorstellen, wie man diesen Prozess allgemein als „gut" oder ,schlecht" bewerten, Wahrscheinlichkeiten für ein Eintreten errechnen oder den Nutzenwert für die Allgemeinheit erfassen könnte. „I do not think that the primary role of ethics is to tell us what is good and bad; rather, it is to force us to raise uncomfortable questions about aspects of the human condition that we ordinarily take for granted.“ (Dupuy 2004, S. 13)

c) Möglicherweise lassen sich nicht-intendierte Folgen im Zuge der Realisierung weiter oben formulierter Erwartungen (einer NBIC-Konvergenz) antizipieren. Das ist sicherlich mit der vorangegangenen Frage der Wahrscheinlichkeit der Realisierung gekoppelt (siehe 1). Wissen wir aber wenig über die Möglichkeit der Realisierung, dann begeben wir uns auf den Weg in Richtung Prognostik oder Spekulation. Das bedeutet wiederum, dass wir uns auf ein mehrstufiges und kaskadenhaftes Kontingenzarrangement ${ }^{4}$ einlassen, da sich Prognosen in der Gegenwart notwendigerweise nur als kontingent beschreiben lassen. ${ }^{5}$ In unserem Fall haben wir es zu tun mit

- Technologien, die möglich gemacht werden (oder nicht);

- der Realisierung von erwünschten Folgen (als intendierte Zwecke oder als Zufälle);

- der Realisierung von unerwünschten Folgen (als nicht intendiert);

- der Unsicherheit, ob nach zukünftigen Maßstäben ähnlich über intendierte/nichtintendierte oder erwünschte/unerwünschte Folgen geurteilt wird, wie wir es gegenwärtig tun. ${ }^{6}$

Insgesamt erscheint eine prospektive Beobachtung von wissenschaftstechnologischen Themen ein recht wackeliger Weg, um zu aussagekräftigen Analysen zu gelangen.

d) Eine vielversprechende Möglichkeit der Interpretation wissenschaftstechnologischer Entwicklungen bietet sich einerseits in einer genaueren Bestimmung kognitiver und normativer Sinngehalte in den korrespondierenden Erwartungskomplexen und andererseits in einer Rekonstruktionen der Entstehung der Themen im Fokus, wie es zum Beispiel im Hinblick auf die Entstehung der Mikrosystemtechnik als „Prozesse der Institutionalisierung“ vorgeführt wird (Bender 2005). In diesem Sinne soll an dieser Stelle eine rekonstruktive Perspektive vorgeschlagen werden, die Zukunftseinschätzungen erst einmal zurückstellt, aber trotzdem auf eventuelle Veränderungen in der Konfiguration von Wissenschaft, Technik, Innovation und Politik aufmerksam machen kann. 


\section{Wissenspolitik als Regulierung?}

In einer ersten Annäherung an den Begriff der Wissenspolitik unterscheiden Bechmann und Stehr zwei Typen von Versuchen: (1) Versuche, die sekundären Folgen von bereits praktisch umgesetztem Wissen zu kontrollieren und (2) Versuche, Wissensansprüche der Wissenschaft von außerhalb der Grenzen des Wissenschaftssystems unmittelbar zu kontrollieren (Bechmann, Stehr 2004, S. 9). Ersteres Argument zielt auf das Thema der Folgenantizipation und -kontrolle und ist damit ein klassisches Regulierungsthema. Letzteres zielt auf einen Begriff der Regulierung als Anstrengung unterschiedlichster Gruppen und Institutionen in der Gesellschaft, die Wissensproduktion zu „disziplinieren“. Das zielt offensichtlich auf einen Eingriff in den Entstehungszusammenhang neuen Wissens.

Die Autoren geben an, dass der Staat in diesem Zusammenhang zwar ein wichtiger, aber nicht der primäre oder alleinig relevante Akteur von Regulierungsmaßnahmen sei. Vielmehr wird mit Wissenspolitik ein übergreifender Komplex bezeichnet, der durch den strategischen Einsatz von politischer und juristischer Herrschaft sowie von ökonomischen Ressourcen und kulturellen Praktiken gekennzeichnet ist und durch den die praktische Realisierung von Wissen zu beeinflussen bzw. zu steuern versucht wird. Anlass solcher Maßnahmen ist immer eine Reaktion auf ,gedachte Folgen“ bestimmter Erkenntnisse (Bechmann, Stehr 2004, S. 9).

\section{Regulierung der Wissensproduktion?}

Wie ist eine Beeinflussung, Steuerung oder gar Regulierung der Wissensproduktion denkbar? Was sind die Voraussetzungen dafür? „Regulierung meint (...) eine Operation, die von der Erwartung geleitet ist, dass ein anderer auf Grund der Beobachtung dieser Operation seinen Zustand ändern werde." (Bora 2002, S. 255) Eine Handlung, die eine Zustandsänderung herbeiführen will, muss aber mit Wissen unterlegt sein. Muss also diejenige Instanz, die diese $\mathrm{Zu}-$ standsänderung herbei führen will, mehr oder besseres Wissen mobilisieren können, als ihr Regulierungsobjekt? Bora argumentiert, dass Regulierung auf Bereiche des Nicht-Kontrollierten oder Nicht-Kontrollierbaren und damit auf Nicht-Wissen oder unvollständigen Wissens trifft. Dieser Bereich muss ,latent" gehalten werden, um einerseits überhaupt entscheiden zu können („Unsicherheitsabsorbtion“ bei March, Simon 1993, S. 186) und die Entscheidung, das andere ihrerseits das Entscheidungsverhalten ändern, realistisch erscheinen zu lassen. Regulierung muss „Sinn“ machen. Es entsteht offenbar eine Spannung aus Entscheidungszwang, die z. B. den Aufgabenbeschreibungen von Regulierungs- oder Verwaltungsorganisationen geschuldet sein kann, und der Begründungsverpflichtung. Boras These lautet: Operationen als Kontrolle machen nur Sinn, wenn Einflussmöglichkeiten auf den zu kontrollierenden Bereich tatsächlich unterstellt werden können. „Wo dies nicht gelingt, können sich Operationen nicht sinnvoll an den Erwartungen externer Zustandsänderung orientieren." (Bora 2002, S. 258) Die Fiktion des „Unter-Kontrolle-Bringen-Könnens“ ist Bedingung der Möglichkeit von Regulierung. Für unser Thema bedeuten diese Thesen, dass

- eine Determinierung des Regulierungsobjekts durch regulatives Handeln verneint wird,

- zwischen der Selbstreferenz der Regulierung und (aus dieser Sicht) einer Fremdreferenz des Regulierungsgegenstands unterschieden werden muss, der wiederum auch selbstreferenziell operiert,

- die „Mittel“ der Regulierung auf beiden Seiten eine realistische Möglichkeit von Einflussnahme erwarten lassen, also einerseits genügend Begründungspotenzial bieten (für die Richtigkeit der Eingriffe in die Autonomie anderer Bereiche) und andererseits eine hinreichende Beschränkung der Autonomie bedeuten (im Falle einer Nicht-Entsprechung).

Für eine Regulierung von CT oder NBIC stellen sich die Fragen, was eigentlich der Regulierungsgegenstand ist? Inwieweit liegen beide wissenschaftstechnologischen Themen der aktuellen Forschung zugrunde? Mit welchen Mittel kann dort eingegriffen werden?

\section{Prozesse der Institutionalisierung}

In den letzten Jahren sind einige techniksoziologische Ansätze formuliert worden, die evolutionäre Prozesse der Technikentwicklung rekonstruieren, indem von der Idee rationaler Planung 
abgesehen und stattdessen von eigendynamischen „structural drifts“ ausgegangen wird. Zu nennen sind einerseits Autoren, die auf die Wirkmächtigkeit von technologischen Versprechungen (van Lente 1993; van Lente 2000) oder von simplifizierenden Selbstbeschreibungen „folk-theories“ (Rip 2006) aufmerksam machen, und andererseits Autoren, die Prozesse der Institutionalisierung beschreiben (Bender 2005; Werle 2005). Allen Ansätzen ist - so meine These - gemeinsam, dass sie die Entstehung von wissenschaftstechnologischen Themen und die Möglichkeit der - zunächst einmal unwahrscheinlichen - Kooperation unterschiedlichster Akteure im weiteren Verlauf der "Setzung“ einer Agenda analysieren. Dabei werden Prozesse der gegenseitigen Beobachtung und des aneinander Aufrichtens als „Koevolution“ von Markt und Hierarchie orientierten Organisationen sowie von Netzwerken beschrieben (Werle 2005, S. 324), die ohne zentrale Steuerungsinstanz auskommen. Insgesamt lassen sich vier aufeinander aufbauende Stadien der Technikentwicklung unterscheiden:

(1) Expectation Statements: Diese beinhalten Erzählungen über vielversprechende Technologien (,,promising technologies" bei van Lente 1993), die sich auf Szenarien zukünftiger technologischer, ökonomischer und sozialer Entwicklungen gründen. Die Realisierung dieser Zukünfte bedarf einer gemeinsamen Anstrengung unterschiedlicher wissenschaftlicher Disziplinen, aber auch von privatwirtschaftlichen Unternehmen und der Politik. Spezielle Anwendungen sind zu diesem Zeitpunkt noch nicht exakt $\mathrm{zu}$ bestimmen. Die Erwartungen sind notwendigerweise übertrieben, um Unterstützung für die Sache zu generieren. Die Wahrscheinlichkeit von Enttäuschungen ist dementsprechend hoch. ${ }^{7}$

(2) Spezifizierung einer wissenschaftlichen Agenda: Unspezifische Versprechen werden aktiv und systematisch in spezifischen Erwartungen gewandelt. Bender spricht von organisierter Antizipation, wenn im Folgenden in den Wissenschaften selbst Zielrichtungen, Aufgaben und kritische Variablen (Innovationspotenzial, Kosten, Zeiträume) für die Technologieentwicklung genauer spezifiziert werden (Bender 2005, S. 181). Mit Hinweis auf die Lösung politischer und wirtschaftlicher Probleme (z. B. die Konkurrenzfähigkeit der regionalen Industrie) werden Bedingungen für die Herstellung kognitiver Objekte (wissenschaftliches Wissen) formuliert.

(3) Spezifizierung einer politischen Agenda: Die organisierte Antizipation auf der Forschungsseite hat Konsequenzen auf der Förder- und Anwendungsseite. Eine Spezifizierung der technologischen Entwicklung beinhaltet eine bestimmte Zukunft, die zu gestalten ist. Daran können sich Förderpolitik oder Investitionen orientieren. Politische Akteure formulieren Forschungsprogramme, in denen wiederum spezifiziert wird, unter welchen Bedingungen Forschung alimentiert wird. Aus kognitiven Erwartungen zur Lösung wissenschaftlicher Probleme werden dann normative Erwartungen zur Lösung von politischen Problemen (erfolgreiche Innovationspolitik).

(4) Institutionalisierung eines wissenschaftlichen oder technologischen Paradigmas: Kennzeichen für die Etablierung eines wissenschafts-technologischen Paradigmas ist ein Rahmen von akzeptierten Theorien, Methoden oder Anwendungen. Ein Paradigma setzt Standards für wissenschaftliche Gültigkeit bzw. Güte und organisiert sowie kanalisiert neue Ideen und Argumente. Ein Paradigma ist relativ stabil. Es ist zwar weiterhin offen für Diskussionen, Kritik und Veränderung, enthält aber auch ein Moment der Irreversibilität in Form eines „Stands der Technik“, hinter den nicht zurückgegangen werden kann (Bender 2005, S. 183f.). Weiterer Ausdruck ist die Verwendung einer eigenen Sprache mit einem spezifischen Fachvokabular, eigene Veröffentlichungsmedien (spezialisierte Zeitschriften und Veranstaltungen) sowie die Etablierung eigener Ausbildungscurricula.

\section{Die Institutionalisierung von NBIC}

Wenn wir uns an diese Differenzierung von Stufen der Institutionalisierung anlehnen und für die Themen CT und speziell NBIC übernehmen, dann lässt sich kontrollierter einschätzen, mit welchem Regulierungsgegenstand wir es zu tun haben. Die Frage lautet dann, in wel- 
chem Stadium der Institutionalisierung befindet sich das Thema NBIC eigentlich? Als Extrakt aus zahlreichen Dokumentenstudien und Expertengesprächen im Projekt Knowledge NBIC lassen sich (wiederum in aller Kürze) folgende Aussagen destillieren:

$\mathrm{Zu}$ (1): Unbestritten ist die Existenz von wirkmächtigen expectation statements. Der Bericht der beiden Autoren Mihail C. Roco und William S. Bainbridge (2002) wurde zu Beginn bereits erwähnt und ist Objekt zahlloser Kommentierungen geworden. Dazu kam im Jahre 2004 ein - wie ich es nenne - counter expectation statement als Ergebnis der Beratungen einer high level expert group (HLEG) „Foresighting the New Technology Wave", die einen umfangreichen und ebenso umfänglich kommentierten Bericht mit dem Titel „Converging Technologies - Shaping the Future of European Societies" vorgelegt hat (Nordmann 2004). Zu den diversen Erwartungen über die möglichen technologischen Entwicklungen, die durch ein Konvergieren von bereits existierenden Wissensdomänen initiiert werden sollen, ist hinlänglich berichtet worden. Festzuhalten gilt aber die starke Aufmerksamkeit, die dem Thema „Interdisziplinarität in der Forschung" in beiden Berichten gewidmet wurde. Beispiele aus 2002 sind:

- „...interdisciplinary research projects designed to promote technological convergence..." (Roco, Brainbridge 2002, S. 14);

- „Interdisciplinary education programs, especially in graduate school, can create a new generation of scientists and engineers who are comfortable working across fields and collaborating with colleagues from a variety of specialties" (dies. 2002, S. 8);

- „interdisciplinary journals, periodic new conferences, and formal partnerships between professional organizations must be established" (dies. 2002, S. 9).

In dem Bericht der HLEG wird diese Idee weiter getrieben und es werden der NBICKonvergenz weitere wissenschaftliche Disziplinen als Anreicherung zugedacht: „Nano-BioInfo-Cogno-Socio-Anthro-Philo-Geo-Eco-UrboOrbo-Macro-Micro-Nano“ lautet die Formel. Alles in allem soll daraus eine genuin europäische Variante des NBIC-Konzepts entstehen: "Converging Technologies for the European Knowledge Society (CTEKS)“.
Zu (2) Zahlreiche Gespräche mit Experten und Beobachtung in unterschiedlichen Ländern der EU, die im Rahmen des Projekts „Knowledge NBIC" durchgeführt wurden, haben nicht erkennen lassen, dass eine NBIC-Konvergenz auf der Ebene wissenschaftlicher Forschung stattfindet. Die befragten Forscher und der Forschung nahestehende Beobachter konnten mit dem Label NBIC wenig anfangen, schon gar nicht bezeichneten sie selbst ihre Forschung in dieser Art; auch hatten viele der Befragten Schwierigkeiten, Konvergenz und Interdisziplinarität zu unterscheiden. Es lässt sich aus den Beobachtungen festhalten, dass keine Anzeichen für die Etablierung einer NBIC-Agenda in den Wissenschaften zu erkennen waren, so wie man es etwa in der Mikrosystemtechnik oder in der Nanotechnologie könnte.

$\mathrm{Zu}$ (3) Im Gegensatz dazu lassen sich aber Anzeichen für die Etablierung einer politischen Agenda hinsichtlich von CT und NBIC entdecken, und zwar in Form organisatorischer und programmatischer (Um-)Orientierungen. Die EU-Administration hat beispielsweise im ,Directorate-General for Research - Directorate G: Industrial Technologies" die Einheit G.4: „Nano- and Converging Sciences and Technologies" benannt. Das deutsche Ministerium für Bildung und Forschung erwähnt in ihrer „Nano-Initiative - Aktionsplan 2010“ die Idee der konvergierenden Technologien, wobei die Erwartungshaltung sich hier auf Innovationen hinsichtlich von Heilungs- und Therapiemöglichkeiten in der Medizin und Medizintechnik richten. ${ }^{8}$ Aber auch in Förderprogrammen taucht die Idee der Konvergenz als Innovationsmotor auf. Beispielsweise geschieht dies in einer Bekanntmachung des BMBF im Innovationsbereich „Systemintegration“ im Rahmenprogramm Mikrosysteme (2004-2009) Richtlinien zur Förderung im thematischen Schwerpunkt „Mikro-Nano-Integration für die Mikrosystemtechnik (MNI-mst)“: „Aufgrund der komplexen Herausforderung
wird in diesem Zusammenhang die Interdis-
ziplinarität bzw. die Konvergenz von Techno-
logien eine wichtige Rolle spielen. Durch die
Konvergenz von Spitzentechnologien, wie
z. B. NBIC (Nanotechnologie, Biotechnolo-
gie, Informationstechnik, Kognitionswissen-
schaften), werden neue Möglichkeiten des
wissenschaftlich-technologischen Fortschritts
und wirtschaftlichen Wachstums erwartet. ${ }^{\circ 9}$ 
Hier werden offensichtlich normative Erwartungen formuliert, die auf die Organisation der Wissensproduktion abzielen: Es werden Bedingungen der Forschungsförderung benannt, an denen man sich orientieren soll, wenn man Förderung erhalten will. Antragsteller müssen ihre Forschung so organisieren, dass Formen der interdisziplinären Forschung sichtbar werden.

\section{Interdisziplinarität als innovations- förderndes Leitbild}

Die Betonung von Interdisziplinarität trifft den Kern aktueller wissenschaftssoziologischer Debatten. Protagonisten der ,new production of knowledge" behaupten eine Verschiebung von streng disziplinärer Forschung in Richtung inter- und transdisziplinärer Kooperation (Gibbons et al. 1994, S. 147ff.). ${ }^{10}$ Was ist für unseren Fall dahingehend zu erwarten? Eine Differenzierung des Wissenschaftsbetriebs in Disziplinen hat sich aufgrund der Verdichtung und Abgrenzung von Innovationschancen als auBerordentlich erfolgreiche Organisationsweise erwiesen. Dies hat offensichtlich immer dann negative Konsequenzen, wenn eine Abdichtung der einzelnen Disziplinen verhindert, dass „übergeordnete Fragen“ in den Blick genommen werden, konstatiert Luhmann (Luhmann 1994, S. 456). Neuerdings werden die Innovationschancen disziplinärer Forschung vehement bestritten und gebetsmühlenartig argumentiert, nur interdisziplinäre Forschung sei erfolgversprechend. Interdisziplinarität kondensiert zum „,inviolate level“ der Innovationspolitik (ebenso die Idee Vernetzung in „Kompetenznetzwerke oder -cluster"): Interdisziplinarität gerinnt zum Eigenwert (als Entscheidungsprämisse), die nur noch mit sehr hohem Begründungsaufwand in Frage gestellt werden kann. Bei Luhmann findet sich eine klare Unterscheidung von drei Formen der Interdisziplinarität:

a) „okkasionelle Interdisziplinarität“: Darunter wird verstanden, dass Disziplinen aus Kontakten mit anderen Disziplinen per Zufall lernen können. Es kommt unter Umständen zur Übernahme von Begriffen mit überraschenden Konsequenzen. Dort ist die Fähigkeit der Umwandlung von Überraschungen in Strukturen gefordert. Mittlerweile werden für diese Art des eher unsystematischen Kontakts zahl- reiche Anlässe geschaffen (Tagungen, Projekte, Zeitschriften, Institute).

b) ,temporäre Interdisziplinarität": In zeitlich begrenzten Projekten werden Kooperationen verschiedener Disziplinen „problembezogen" organisiert. Eine Zielerreichung (Problemlösung?) wird angepeilt und die Kooperation auch wieder beendet.

c) „Transdisziplinarität“: Transdisziplinäre Unternehmungen arbeiten von einem gemeinsamen wissenschaftlichen Paradigma aus, das sich von den einzelnen Disziplinen unterscheidet, das aber für mehr als eine Disziplin relevant ist (Luhmann 1994, S. $457 \mathrm{ff}$.).

Genau an diesem Punkt hakt es, wenn man Angaben über die vierte Stufe der Institutionalisierung (Institutionalisierung eines Paradigmas) machen möchte. Es ist auf den ersten Blick nicht erkennbar, welches wissenschaftliche Paradigma die oben angesprochenen Wissensdomänen der Nano-, Bio-, Info-Technologien in Kombination mit den Kognitionswissenschaften kognitiv integriert? Die expectation statements fordern, dass die institutionellen und organisatorischen Bedingungen dafür geschaffen werden, geben aber keine Antwort, wie eine gemeinsam geteilte wissenschaftliche Problemstellung aussieht. „Innovation“ und „human, social or societal enhancements" sind sicherlich keine wissenschaftlichen Probleme, wie es zum Beispiel „Prozesse der Selbstorganisation“ oder „Abweichungen verstärkende Effekte" wären, sondern allein innovationspolitische Probleme. Am Ende könnte sich die ganze Rhetorik um Konvergenz und NBIC als völlig losgelöst von den Vorgängen an der Wissenschaftsfront erweisen, wie z. B. auch Schummer (2009) betont.

Das hat zweierlei Konsequenzen für „Wissenspolitik". Normative Orientierungen, die sehr frühzeitig im Zuge der Wissensproduktion mögliche negative Konsequenzen der Technologieentwicklung erkennen und regulieren wollen, bekommen im Fall von NBIC ihren Gegenstand gar nicht zu fassen, weil dieser noch nicht - oder noch nicht absehbar - als wissenschaftliche Agenda institutionalisiert ist. Normative Orientierungen, die eine andere Organisation der Wissensproduktion fordern, wiederholen "leere" Erfolgsformeln, weil sie ihrerseits die Bedingungen inter- und transdisziplinärer Forschung nicht beeinflussen können, die da lauten: die 
Generierung gemeinsam geteilter wissenschaftlicher Problemstellungen und Paradigmen.

\section{Schluss}

Inwieweit CT und NBIC zukunftsträchtige wissenschaftstechnologische Themen sind, das soll an dieser Stelle offen bleiben. Das mögen andere beurteilen. Interessant ist die Beobachtung, wie im Prozess der Institutionalisierung von CT und NBIC eine politische Agenda die einer wissenschaftlichen überholt hat, und wie aus kognitiven irgendwann normative Erwartungen werden: Dies geschieht, wenn politisch Bedingungen für die Organisation wissenschaftlicher Erkenntnisproduktion gesetzt werden. Hier erlangt die Idee des, from promise to requirement" (van Lente 2000) eine ganz neue Bedeutung. Dabei ist auch nicht auszuschließen, dass in naher Zukunft eine entsprechende wissenschaftliche Agenda aufholt.

\section{Anmerkungen}

1) Vgl. http://www.converging-technologies.org

2) Die nachfolgenden Überlegungen verdanken sich zahlreichen Diskussionen mit Gotthard Bechmann und Simon Pfersdorf. Die nachfolgenden Besprechungen spiegeln in keinem Fall einen methodologischen oder konzeptionellen Konsens innerhalb des Projekt-Konsortiums wider.

3) Zu finden in Roco, Bainbridge 2002, S. $35 \mathrm{ff}$.

4) Kontingenz wird hier verstanden als Verneinung von Unmöglichkeit und Notwendigkeit.

5) Dabei gehen wir prinzipiell von einer „offenen Zukunft" aus, die wir noch nicht kennen, auf die wir keinen Zugriff haben, die wir nur in der Gegenwart beschreiben können: Dies wird dann in Form von gegenwärtigen Zukünften als Prognose begriffen, die sich dann als zukünftige Gegenwart manifestiert. Prognosen oder Spekulationen wie diese können sich dann als ,zutreffend“" oder „,nicht zutreffend“ erweisen.

6) Es ließe sich noch eine weitere Stufe beschreiben: Wenn, zusätzlich zur Beschreibung möglicher Folgen, Vorschläge zum vorsorglichen Handeln erarbeitet werden sollen, die als Grundlagen für gegenwärtiges Entscheiden herhalten, dann gilt es den Einfluss der Prognose auf das Prognostizierte mit zu berücksichtigen. Denn davon würde auch abhängen, was hinsichtlich der Ausgangsfrage der Fall sein wird, nämlich, ob bestimmte Technologien realisiert werden oder nicht (siehe dazu auch Koselleck 1985, S. 45f., und zur „Entworfenen Zeit“ bei Dupuy 2005).

7) Siehe dazu das Konzept des „hype-disappointment-cycles" (Rip 2006, S. 352).

8) Siehe http://www.bmbf.de/pub/nano_initiative_a ktionsplan_2010.pdf, S. 28, download 4.6.2009

9) Siehe http://www.mstonline.de/foerderung/innov aber/bekanntmachung_mni.pdf, S. 1, download 4.6.2009

10) Ein Argument, das an anderer Stelle als empirisch nicht haltbar beschreiben wird (vgl. z. B. Krücken 2001).

\section{Literatur}

Bechmann, G.; Stehr, N., 2004: Wissenspolitik - ein neues Forschungs- und Handlungsfeld? In: Technikfolgenabschätzung - Theorie und Praxis 13/3 (2004), S. 5-20

Bender, G., 2005: Technologieentwicklung als Institutionalisierungsprozess. In: Zeitschrift für Soziologie 34/3 (2005), S. 170-186

Bora, A., 2002: Ökologie der Kontrolle. Technikregulierung unter der Bedingung von Nicht-Wissen. In: Engel, Chr.; Halfmann, J.; Schulte, M. (Hg.): Wissen, Nichtwissen, unsicheres Wissen. BadenBaden, S. 253-275

Coenen, Chr., 2008: Konvergierende Technologien und Wissenschaften. Der Stand der Debatte und politische Aktivitäten zu „Converging Technologies"; http://www.tab.fzk.de/de/hp.htm (download 4.9.09)

Coenen, Chr.; Rader, M.; Fleischer, T., 2004: Of Visions, Dreams and Nightmares: The Debate on Converging Technologies. Report on the Conference „Converging Technologies for a Diverse Europe“. In: Technikfolgenabschätzung in Theorie und Praxis 13/3 (2004), S. 118-125

Dupuy, J.-P., 2004: Complexity and Uncertainty. A Prudential Approach to Nanotechnology; http://portal .unesco.org/ci/en/files/20003/11272944951Dupuy2.p df/Dupuy2.pdf (download 28.1.2009)

Dupuy, J.-P., 2005: Aufgeklärte Unheilsprophezeiungen. Von der Ungewissheit zur Unbestimmbarkeit technischer Folgen. In: Gamm, G.; Hetzel, A. (Hg.): Unbestimmtheitssignaturen der Technik: eine neue Deutung der technisierten Welt. Bielefeld, S. 81-102

Gibbons, M.; Limoges, C.; Nowotny, H. et al., 1994: The New Production of Knowledge: The Dynamics of Science and Research in Contemporary Societies. London 
Koselleck, R., 1985: Die unbekannte Zukunft und die Kunst der Prognose. In: Lutz, B. (Hg.): Soziologie und gesellschaftliche Entwicklung: Verhandlungen des 22. Deutschen Soziologentages in Dortmund 1984. Frankfurt a. M, S. 45-59

Krücken, G., 2001: Wissenschaft im Wandel? Gegenwart und Zukunft der Forschung an deutschen Hochschulen. In: Stölting, E.; Schimank, U. (Hg.): Die Krise der Universitäten. Wiesbaden, S. 326-345

Luhmann, N., 1994: Die Wissenschaft der Gesellschaft. Frankfurt a. M., 2. Aufl.

March, J.G.; Simon, H., 1993: Organizations. Cambridge, 2. Aufl.

Nordmann, A. (Rapporteur) (Hg.), 2004: Converging Technologies - Shaping the Future of European Societies. HLEG - High Level Expert Group "Foresighting the New Technology Wave", Brussels

Rammert, W., 2003: Zwei Paradoxien einer innovationsorientierten Wissenspolitik: Die Verknüpfung heterogenen und die Verwertung impliziten Wissens. In: Soziale Welt 54 (2003), S. 483-408

Rip, A., 2006: Folk Theories of Nanotechnologists. In: Science as Culture 15/4 (2006), S. 349-365

Roco, M.C.; Bainbridge, W.S., 2002: Converging Technologies for Improving Human Performance: Nanotechnology, Biotechnology, Information Technology and Cognitive Science; http://www.wtec.org/ ConvergingTechnologies/Report/NBIC-report.pdf (download 7.9.09)

Stehr, N., 2003: Wissenspolitik: die Überwachung des Wissens. Frankfurt a. M.

van Lente, H., 1993: Promising technology: the dynamics of expectations in technological developments. Delft

van Lente, H., 2000: Forceful Futures: From Promise to Requirement. In: Brown, N.; Rappert, B.; Webster, A. (Hg.): Contested Futures: A Sociology of Prospective Techno-science. Aldershot u. a., S. 43-63

Wehling, P., 2006: Im Schatten des Wissens? Perspektiven der Soziologie des Nichtwissens. Konstanz Werle, R., 2005: Institutionelle Analyse technischer Innovation. In: Kölner Zeitschrift für Soziologie und Sozialpsychologie 57/2 (2005), S. 308-332

\section{Kontakt}

Dr. phil. Christian Büscher Forschungszentrum Karlsruhe ITAS

Postfach 36 40, 76021 Karlsruhe

Tel.: +49 (0) 7247 / 82 - 3181

E-Mail: buescher@itas.fzk.de 